\title{
The dynamics of COVID-19 in the UAE based on fractional derivative modeling using Riesz wavelets simulation
}

\author{
Mutaz Mohammad $^{1 *}$ (D), Alexander Trounev ${ }^{2}$ and Carlo Cattani ${ }^{3}$
}

\section{"Correspondence:}

Mutaz.Mohammad@zu.ac.ae 'Zayed University, Abu Dhabi, United Arab Emirates

Full list of author information is available at the end of the article

\section{Springer}

\begin{abstract}
The well-known novel virus (COVID-19) is a new strain of coronavirus family, declared by the World Health Organization (WHO) as a dangerous epidemic. More than 3.5 million positive cases and 250 thousand deaths (up to May 5, 2020) caused by COVID-19 and has affected more than 280 countries over the world. Therefore studying the prediction of this virus spreading in further attracts a major public attention. In the Arab Emirates (UAE), up to the same date, there are 14,730 positive cases and 137 deaths according to national authorities. In this work, we study a dynamical model based on the fractional derivatives of nonlinear equations that describe the outbreak of COVID-19 according to the available infection data announced and approved by the national committee in the press. We simulate the available total cases reported based on Riesz wavelets generated by some refinable functions, namely the smoothed pseudosplines of types I and II with high vanishing moments. Based on these data, we also consider the formulation of the pandemic model using the Caputo fractional derivative. Then we numerically solve the nonlinear system that describes the dynamics of COVID-19 with given resources based on the collocation Riesz wavelet system constructed. We present graphical illustrations of the numerical solutions with parameters of the model handled under different situations. We anticipate that these results will contribute to the ongoing research to reduce the spreading of the virus and infection cases.
\end{abstract}

Keywords: Fractional differential equations; Novel coronavirus; Riesz wavelet system; Smoothed pseudosplines; Mathematical model

\section{Introduction}

In March 2020, WHO has announced the novel coronavirus as a pandemic after the outbreak on the end of January 2020, when it was declared a public health emergency for the global. Since then, the pandemic has affected almost all countries around the world and killed more than 290,000 of people worldwide. The virus can easily spread from one to another, and no treatment or vaccine can do the needs $[1,2]$. Even though a vaccine could be more than a year away, doctors are experimenting with drugs and therapies to help ease the virus symptoms/spreading.

(c) The Author(s) 2021. This article is licensed under a Creative Commons Attribution 4.0 International License, which permits use, sharing, adaptation, distribution and reproduction in any medium or format, as long as you give appropriate credit to the original author(s) and the source, provide a link to the Creative Commons licence, and indicate if changes were made. The images or other third party material in this article are included in the article's Creative Commons licence, unless indicated otherwise in a credit line to the material. If material is not included in the article's Creative Commons licence and your intended use is not permitted by statutory regulation or exceeds the permitted use, you will need to obtain permission directly from the copyright holder. To view a copy of this licence, visit http://creativecommons.org/licenses/by/4.0/. 
Given the dangerous situation, many researchers started working on formulating models that best describe the dynamics of all possible parameters responsible for the daily cases reported including deaths, control the fatality rate, and prediction of COVID-19 behavior in future within a specific region. For example, Alberto [3] has developed a mathematical model to identify the number of days students could attend school to allow them a better learning experience while mitigating infections of COVID-19.

It is known that several models can describe a specific system, which is a challenging step. However, in this paper, we use the well-known parsimony principle, where the model should be constructed in a simple way as possible but also with complexity when needed.

Fractional derivatives have been proven to be a useful tool in a wide area of applications in science and engineering [4-15], including customary in groundwater analysis, the modeling of infection disease, and epidemic systems to discover and predict the spreading of many diseases. It is known that Covid-19 originated in bats and infected humans and can infect several animals such as cats and ferret. There is no case (up to date) of direct transmission from a bat to human, but yet a proposal says that there is a host-reservoir most likely involved between them. We consider the model presented in [16] developed based on

$$
\text { Bats } \Rightarrow \text { Hosts } \Rightarrow \text { Reservoir } \Rightarrow \text { People }
$$

formulation setting in terms of the Atangana-Baleanu fractional derivative. In this paper, we use the Caputo fractional derivative definition to study the model. The advantage of using this definition is that it allows traditional and various types of ICs in creation of a dynamical model. Wavelets appear in a variety of advanced applications such as filter bank constructions arising in image processing. This is largely due to the fact that wavelets have the right structure to capture the sparsity in "physical" images, perfect mathematical properties such as its multiscale structure, sparsity, smoothness, compact support, and high vanish moments. It has many applications in fractional integral and differential equations (see, e.g., [17-30]).

Riesz wavelets in $L_{2}(\mathbb{R})$ have been extensively used in the context of both pure and numerical analysis in many applications due to their well prevailing and recognized theory and their natural properties such as sparsity and stability, which lead to a well-conditioned scheme. In this paper, we present an effective and accurate technique based on Riesz wavelets for solving the transmission model of COVID-19 based on the Caputo fractional derivative. The advantage of such wavelets lies on their simple structure in the reduced systems and in the powerfulness of obtaining approximated solutions for equations that have weakly singular kernels. The proposed method shows a good performance and high accuracy orders.

Let us recall some definitions and notation. A function $\phi \in L_{2}(\mathbb{R})$ is called refinable if

$$
\phi=\sum_{k \mathbb{Z}} a[k] \phi(2 \cdot-k)
$$


where $a[k] \in \ell_{2}(\mathbb{Z})$ is finitely supported sequence, called the refinement mask of $\phi$. The corresponding wavelet function is defined by

$$
\psi=\sum_{k \mathbb{Z}} b[k] \phi(2 \cdot-k)
$$

where $b[k] \in \ell_{2}(\mathbb{Z})$ is finitely supported sequence, called the high pass filter of $\psi$.

In this paper, for $f \in L_{1}(\mathbb{R})$ (which can be extended to $L_{2}(\mathbb{R})$ ), we use the Fourier transform

$$
\hat{f}(\xi)=\frac{1}{\sqrt{2 \pi}} \int_{\mathbb{R}} e^{-i x \xi} f(x) d x
$$

The Fourier series of the sequence $a$ is defined as

$$
\hat{a}(\xi)=\sum_{k \in \mathbb{Z}} a[k] e^{-i k \xi}, \quad \xi \in \mathbb{R} .
$$

Pseudosplines have attracted many researchers due to their significant contribution to both numerical computations and analysis. The constructions of pseudosplines track back to the well-known work by Daubechies et al. [31,32], It is a family of refinable functions with compact support and has extensive flexibility in wavelets and applications. Pseudosplines are known as a generalization of many well-known refinable functions such as Bsplines, interpolated, and orthonormal refinable functions [33]. We refer the reader to $[31,32,34-38]$ and references therein for more detail.

\section{Riesz wavelets via smoothed pseudosplines}

We use the smoothed pseudosplines introduced in [38] to construct Riesz wavelets and use them to apply our numerical scheme for solving different types of FIDEs. Pseudosplines of order $(p, q)$ of types I and II, ${ }_{k} \phi_{(p, q)}, k=1,2$, are defined in terms of their refinement masks, where

$$
\left|\hat{a}_{(p, q)}(\xi)\right|^{2}=\sum_{m=0}^{q}\left(\begin{array}{c}
p+q \\
m
\end{array}\right)(\cos (\xi / 2))^{2(p+q-m)} \sin ^{2 m}(\xi / 2),
$$

and

$$
{ }_{2} \hat{a}_{(p, q)}(\xi)=\left|{ }_{1} \hat{a}_{(p, q)}(\xi)\right|^{2}
$$

Note that the refinement mask of the pseudosplines of type I of order $(p, q)$ is obtained using the Fejér-Riesz theorem. The refinable pseudospline function generated using the above refinement masks is defined by

$$
{ }_{k} \hat{\phi}_{(p, q)}(\cdot)=\prod_{m=1}^{\infty}{ }_{k} \hat{a}_{(p, q)}\left(\cdot / 2^{m}\right), \quad k=1,2 .
$$

They are two types of smoothed pseudosplines defined by its refinable masks. For $r \geq p$, we have the smoothed refinable pseudosplines of type I $(k=1)$ and II $(k=2)$ of order 
$(r, p, q)$ such that

$$
{ }_{k} \phi_{(r, p, q)}(\cdot)={ }_{k} \phi_{p, q} * \chi_{\left[-\frac{1}{2}, \frac{1}{2}\right]}^{r-p}(\cdot), \quad k=1,2,
$$

where

$$
\chi_{\left[-\frac{1}{2}, \frac{1}{2}\right]}^{r-p}(\cdot)=\chi_{\left[-\frac{1}{2}, \frac{1}{2}\right]} * \cdots * \chi_{\left[-\frac{1}{2}, \frac{1}{2}\right]}, \quad \text { for }(r-p) \text {-times, }
$$

where $\chi_{A}$ is the indicator function of a set $A$. Similarly, the refinement masks of both types of ${ }_{k} \phi_{(r, p, q)}$ for $k=1,2$, respectively, are given by

$$
\left|\hat{a}_{(r, p, q)}(\xi)\right|^{2}=\sum_{m=0}^{q}\left(\begin{array}{c}
p+q \\
m
\end{array}\right)(\cos (\xi / 2))^{2(r+q-m)} \sin ^{2 m}(\xi / 2)
$$

and, for $r \geq 2 p$,

$$
{ }_{2} \hat{a}_{(r, p, q)}(\xi)=\sum_{m=0}^{q}\left(\begin{array}{c}
p+q \\
m
\end{array}\right)(\cos (\xi / 2))^{2 q+r-p} \sin ^{2 m}(\xi / 2) .
$$

Riesz wavelets have been extensively studied in the literature; see, for example, [39] and other references.

Definition 2.1 We say that the set $\mathcal{M}\left(\psi^{\ell}\right)=\left\{\psi_{j, k}^{\ell}=2^{j / 2} \psi^{\ell}\left(2^{j} \cdot-k\right), \ell=1, \ldots, N\right\}, \psi^{\ell} \in$ $L_{2}(\mathbb{R})$, generates a Riesz wavelet in $L_{2}(\mathbb{R})$ if for any finitely supported sequence $\left\{n_{j, k}^{\ell}, \ell=\right.$ $1, \ldots, N ; j, k \in \mathbb{Z}\}$, there exist positive numbers $c$ and $C$ such that

$$
c \sum_{\ell=1}^{N} \sum_{j \in \mathbb{Z}} \sum_{k \in \mathbb{Z}}\left|n_{j, k}^{\ell}\right|^{2} \leq\left\|\sum_{\ell=1}^{N} \sum_{j \in \mathbb{Z}} \sum_{k \in \mathbb{Z}} n_{j, k}^{\ell} \psi_{j, k}^{\ell}\right\|^{2} \leq C \sum_{\ell=1}^{N} \sum_{j \in \mathbb{Z}} \sum_{k \in \mathbb{Z}}\left|n_{j, k}^{\ell}\right|^{2}, \quad \forall g \in L_{2}(\mathbb{R}),
$$

where

$$
\|g\|^{2}=\langle g, g\rangle, \quad \text { and } \quad\langle f, g\rangle=\int_{\mathbb{R}} f(x) \overline{g(x)} d x .
$$

If $\mathcal{M}$ in Definition 2.1 is a Riesz wavelet for $L_{2}(\mathbb{R})$, then we have the following expansion for any function $f \in L_{2}(\mathbb{R})$ :

$$
f=\sum_{\ell=1}^{N} \sum_{j, k \in \mathbb{Z}}\left\langle f, \psi_{j, k}^{\ell}\right\rangle \psi_{j, k}^{\ell}
$$

Equation (2.4) can be truncated by

$$
\mathcal{V}_{M} f=\sum_{\ell=1}^{N} \sum_{j \leq M-1} \sum_{k \in \mathbb{Z}}\left\langle f, \psi_{j, k}^{\ell}\right\rangle \psi_{j, k}^{\ell}
$$

Let us provide some examples of Riesz wavelet systems. Note that $\hat{\phi}$ is only implicitly known as an infinite product. We strongly recommend the reader to have a look at Han's 
book [39] (p. 68, Sect. 6) to get a complete picture about how to plot these wavelets and details.

Example 2.1 For $(r, p, q)=(6,2,1)$, we have the following refinable masks:

$$
\begin{aligned}
{ }_{1} \hat{a}_{(6,2,1)}(\xi) & =\frac{1}{2}(\sqrt{3}+1) e^{-\frac{1}{2} 5 i \xi}\left(1+(\sqrt{3}-2) e^{i \xi}\right) \cos ^{3}\left(\frac{\xi}{2}\right), \\
{ }_{1} \hat{b}_{(6,2,1)}(\xi) & =e^{-i \xi} \overline{\hat{a}}_{(6,2,1)}(\xi+\pi)
\end{aligned}
$$

where

$$
\begin{aligned}
& { }_{1} \phi_{(6,2,1)}(2 \cdot)={ }_{1} \hat{a}_{(6,2,1)}(\cdot)_{1} \phi_{(6,2,1)}(\cdot), \\
& { }_{1} \psi_{(6,2,1)}(2 \cdot)={ }_{1} \hat{b}_{(6,2,1)}(\cdot)_{1} \phi_{(6,2,1)}(\cdot) .
\end{aligned}
$$

Then $\mathcal{M}\left({ }_{1} \psi_{(6,2,1)}\right)$ forms a Riesz wavelet system for $L_{2}(\mathbb{R})$. Note that the vanishing moment for the system is 6 .

Example 2.2 For $(r, p, q)=(9,3,2)$, we have the following refinable masks:

$$
\begin{aligned}
{ }_{2} \hat{a}_{(9,3,2)}(\xi) & =\frac{1}{4} \cos ^{10}\left(\frac{\xi}{2}\right)(-156 \cos (\xi)+33 \cos (2 \xi)+127), \\
{ }_{2} \hat{b}_{(9,3,2)}(\xi) & =e^{-i \xi} \overline{\hat{a}_{(9,3,2)}(\xi+\pi)},
\end{aligned}
$$

where ${ }_{1} \hat{a}_{(9,3,2)}(\xi)$ is obtained using the Fejér-Riesz factorization theorem, so

$$
\left|{ }_{1} \hat{a}_{(9,3,2)}(\xi)\right|^{2} \approx{ }_{2} \hat{a}_{(9,3,2)}(\xi)
$$

where

$$
\begin{aligned}
& { }_{2} \phi_{(9,3,2)}(2 \cdot)={ }_{1} \hat{a}_{(9,3,2)}(\cdot)_{1} \phi_{(9,3,2)}(\cdot), \\
& { }_{2} \psi_{(9,3,2)}(2 \cdot)={ }_{1} \hat{b}_{(9,3,2)}(\cdot){ }_{1} \phi_{(9,3,2)}(\cdot) .
\end{aligned}
$$

Then $\mathcal{M}\left({ }_{2} \psi_{(9,3,2)}\right)$ forms a Riesz wavelet system for $L_{2}(\mathbb{R})$. Here we find ${ }_{1} \hat{a}_{(9,3,2)}(\xi)$ numerically; see Fig. 1 . Note that

$$
\left.|| \hat{a}_{(9,3,2)}(\xi)\right|^{2}-{ }_{2} \hat{a}_{(9,3,2)}(\xi) \mid \leq \mathcal{O}\left(10^{-13}\right) .
$$

Definition 2.2 For a real function $u(t)$ where $t, \alpha>0$, and $n \in \mathbb{N}$, we define the following known fractional derivative and integral operator:

- Caputo's fractional derivative CFD,

$$
\mathcal{D}_{*}^{\alpha} u(t)=\frac{1}{\Gamma(n-\alpha)} \int_{0}^{t} \frac{u^{(n)}(x)}{(t-x)^{\alpha+1-n}} d x, \quad n-1<\alpha \leq n .
$$

- Riemann-Liouville fractional integral operator (R-LFI),

$$
\mathcal{I}^{\alpha} u(t)=\frac{1}{\Gamma(\alpha)} \int_{0}^{t} \frac{u(x)}{(t-x)^{1-\alpha}} d x, \quad n-1<\alpha \leq n .
$$



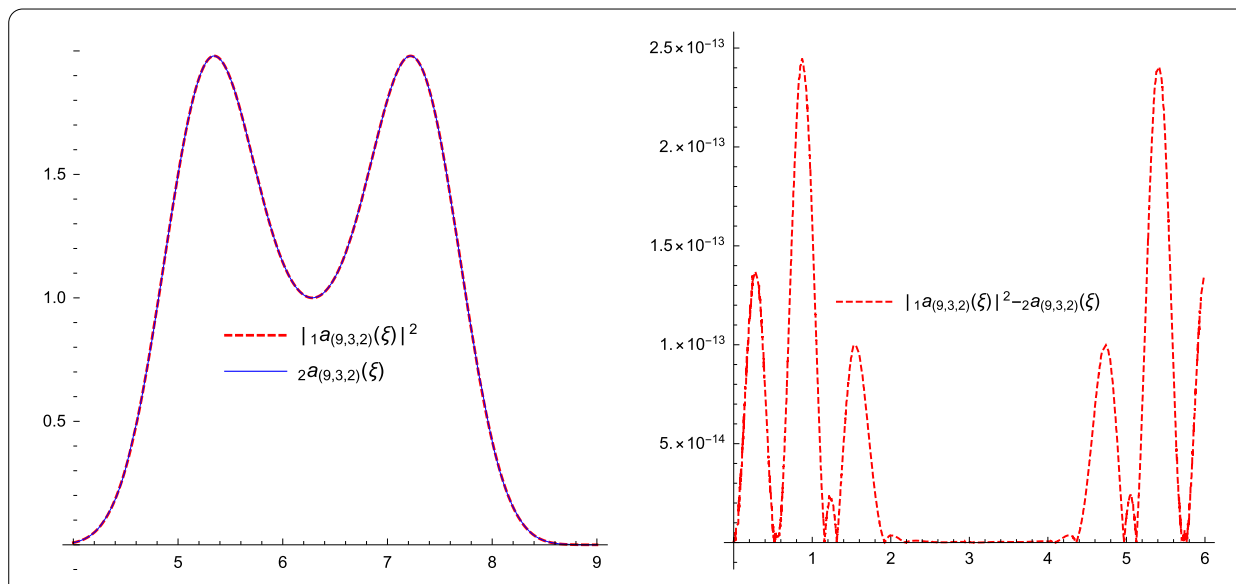

Figure 1 The graphs of the masks $\left.\left.\right|_{1} \hat{a}_{(9,3,2)}(\xi)\right|^{2}$ and $2 \hat{a}_{(9,3,2)}(\xi)$ and their difference in Example 2.2
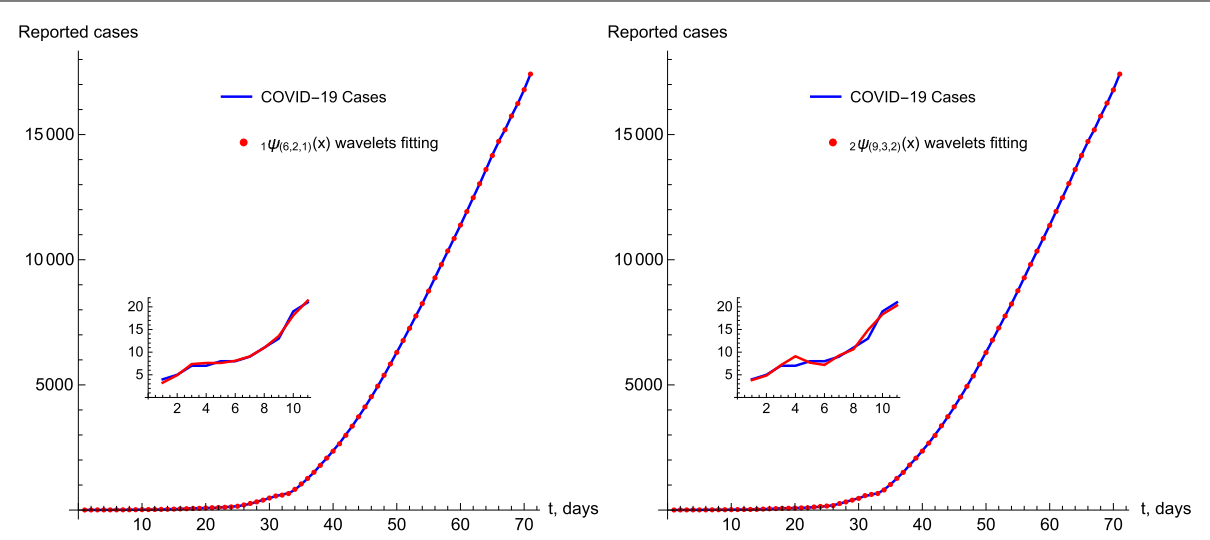

Figure 2 Reported cases of COVID-19 in the UAE with the Riesz wavelet fitting based on Examples 2.1 and 2.2

\section{Transmission model and numerical algorithm based on Riesz wavelet fitting}

The original data are fitted by a set of discrete Riesz wavelet coefficients, where features can be extracted from these coefficients. The simulated data based on the Riesz wavelet systems are illustrated in Fig. 2.

We consider the following new modified transmission model obtained by changing the left-hand side of the system presented in [16] by changing the operator

$$
{ }_{a}^{A B C} \mathcal{D}_{t}^{\alpha} u(t) \quad \text { to } \quad \mathcal{D}_{t}^{\alpha} u(t)
$$

for all unknown functions $S_{p}, E_{p}, I_{p}, A_{p}, R_{p}, M$, where $\rho$ represents the fractional-order parameter. Note that the system is subject to nonnegative initial conditions. So the new system is defined as follows:

$$
\begin{aligned}
& \mathcal{D}_{t}^{\alpha} S_{p}(t)=\Pi_{p}-\mu_{p} S_{p}-\frac{\eta_{p} S_{p}\left(I_{p}+\psi A_{p}\right)}{N_{p}}-\eta_{w} S_{p} M, \\
& \mathcal{D}_{t}^{\alpha} E_{p}(t)=\frac{\eta_{p} S_{p}\left(I_{p}+\psi_{p} A_{p}\right)}{N_{p}}+\eta_{w} S_{p} M-\left(1-\theta_{p}\right) \omega_{p} E_{p}-\theta_{p} \alpha,
\end{aligned}
$$


Table 1 Parameters description and estimated fitted values given $\rho=0.9$

\begin{tabular}{lll}
\hline Parameter & Description & Parameter value \\
\hline$S_{p}(t)$ & The susceptible cases & - \\
$E_{p}(t)$ & The exposed cases & - \\
$l_{p}(t)$ & The infected cases & - \\
$A_{p}(t)$ & The asymptotically cases & - \\
$R_{p}(t)$ & The recovered cases & - \\
$M(t)$ & The infected cases & - \\
$\Pi_{p}$ & Birth rate & 341.706 \\
$\mu_{p}$ & Natural mortality rate & 0.0000353513 \\
$\eta_{p}$ & Contact rate & 0.01 \\
$\psi_{p}$ & Transmissibility multiple & 0.01 \\
$\eta_{w}$ & Disease transmission coefficient & 0.000001 \\
$\theta_{p}$ & The proportion of asymptomatic infection & 0.09 \\
$\omega_{p}$ & Incubation period (bats) & 0.00039 \\
$\rho_{p}$ & Incubation period (hosts) & 0.001 \\
$\tau_{p}$ & recovery rate of $I_{p}$ & 0.1593 \\
$\tau_{a b}$ & recovery rate of $A_{p}$ & 0.95 \\
$\varrho_{p}$ & Contribution of the virus to $M$ by $I_{p}$ & 0.0001 \\
$\varpi_{p}$ & Contribution of the virus to $M$ by $A_{p}$ & 0.00089 \\
$\pi$ & Removing rate of virus from $M$ & 0.009 \\
\hline
\end{tabular}

$$
\begin{aligned}
& \mathcal{D}_{t}^{\alpha} I_{p}(t)=\left(1-\theta_{p}\right) \omega_{p} E_{p}-\left(\tau_{p}+\mu_{p}\right) I_{p}, \\
& \mathcal{D}_{t}^{\alpha} A_{p}(t)=\theta_{p} \rho_{p} E_{p}-\left(\tau_{a p}+\mu_{p}\right) A_{p}, \\
& \mathcal{D}_{t}^{\alpha} R_{p}(t)=\tau_{p} I_{p}+\tau_{a p} A_{p}-\mu_{p} R_{p}, \\
& \mathcal{D}_{t}^{\alpha} M(t)=\varrho_{p} I_{p}+\varpi_{p} A_{p}-\pi M .
\end{aligned}
$$

Given the model parameters and its values in Table 1, it is reasonable to consider this model with proper changes as it is formulated based on the resources and cases detected in Wuhan, China. Since the cases are spread to more than 280 countries around the world, some parameter values will be considered in the current study. For more detail about the formulation and stability results, we refer to [16]. To illustrate the fitting, we use some examples of Riesz wavelet systems to be used in the data fitting using the discrete Riesz wavelet transform defined in (1.3) based on different types of smoothed pseudosplines of orders I and II.

We provide a numerical algorithm based on the collocation method by discretizing the domain function across the Riesz wavelet system used to solve the model. The system is generated using the smoothed pseudosplines of types I and II with different orders. The model defined in Equations (3.1)-(3.5) can be reduced as follows:

$$
\begin{aligned}
& \frac{1}{\Gamma(1-\alpha)} \int_{0}^{t} \frac{S_{p}^{\prime}(x)}{(t-x)^{\alpha}} d x=\Pi_{p}-\mu_{p} S_{p}-\frac{\eta_{p} S_{p}\left(I_{p}+\psi A_{p}\right)}{N_{p}}-\eta_{w} S_{p} M, \\
& \frac{1}{\Gamma(1-\alpha)} \int_{0}^{t} \frac{E_{p}^{\prime}(x)}{(t-x)^{\alpha}} d x=\frac{\eta_{p} S_{p}\left(I_{p}+\psi A_{p}\right)}{N_{p}}+\eta_{w} S_{p} M-\left(1-\theta_{p}\right) \omega_{p} E_{p}-\theta_{p} \rho_{p} E_{p}, \\
& \frac{1}{\Gamma(1-\alpha)} \int_{0}^{t} \frac{I_{p}^{\prime}(x)}{(t-x)^{\alpha}} d x=\left(1-\theta_{p}\right) \omega_{p} E_{p}-\left(\tau_{p}+\mu_{p}\right) I_{p},
\end{aligned}
$$




$$
\begin{aligned}
& \frac{1}{\Gamma(1-\alpha)} \int_{0}^{t} \frac{A_{p}^{\prime}(x)}{(t-x)^{\alpha}} d x=\theta_{p} \rho_{p} E_{p}-\left(\tau_{a p}+\mu_{p}\right) A_{p}, \\
& \frac{1}{\Gamma(1-\alpha)} \int_{0}^{t} \frac{R_{p}^{\prime}(x)}{(t-x)^{\alpha}} d x=\tau_{p} I_{p}+\tau_{a p} A_{p}-\mu_{p} R_{p}, \\
& \frac{1}{\Gamma(1-\alpha)} \int_{0}^{t} \frac{M^{\prime}(x)}{(t-x)^{\alpha}} d x=\varrho_{p} I_{p}+\varpi_{p} A_{p}-\pi M .
\end{aligned}
$$

Using collocation method based on the nodes $t_{i}, i \in \mathbb{N}$, in these equations, we obtain the following equations that generate a system of nonlinear equations to be solved numerically:

$$
\begin{aligned}
& -\mathcal{D}_{t}^{\alpha} S_{p}\left(t_{i}\right)+\Pi_{p}-\mu_{p} S_{p}\left(t_{i}\right)-\frac{\eta_{p} S_{p}\left(t_{i}\right)\left(I_{p}\left(t_{i}\right)+\psi A_{p}\left(t_{i}\right)\right)}{N_{p}}-\eta_{w} S_{p}\left(t_{i}\right) M\left(t_{i}\right)=0, \\
& -\mathcal{D}_{t}^{\alpha} E_{p}\left(t_{i}\right)+\frac{\eta_{p} S_{p}\left(t_{i}\right)\left(I_{p}\left(t_{i}\right)+\psi A_{p}\left(t_{i}\right)\right)}{N_{p}} \\
& \quad+\eta_{w} S_{p}\left(t_{i}\right) M\left(t_{i}\right)-\left(1-\theta_{p}\right) \omega_{p} E_{p}\left(t_{i}\right)-\theta_{p} \rho_{p} E_{p}\left(t_{i}\right)=0, \\
& -\mathcal{D}_{t}^{\alpha} I_{p}\left(t_{i}\right)+\left(1-\theta_{p}\right) \omega_{p} E_{p}\left(t_{i}\right)-\left(\tau_{p}+\mu_{p}\right) I_{p}\left(t_{i}\right)=0, \\
& -\mathcal{D}_{t}^{\alpha} A_{p}\left(t_{i}\right)+\theta_{p} \rho_{p} E_{p}\left(t_{i}\right)-\left(\tau_{a p}+\mu_{p}\right) A_{p}\left(t_{i}\right)=0, \\
& -\mathcal{D}_{t}^{\alpha} R_{p}\left(t_{i}\right)+\tau_{p} I_{p}\left(t_{i}\right)+\tau_{a p} A_{p}\left(t_{i}\right)-\mu_{p} R_{p}\left(t_{i}\right)=0, \\
& -\mathcal{D}_{t}^{\alpha} M\left(t_{i}\right)+\varrho_{p} I_{p}\left(t_{i}\right)+\varpi_{p} A_{p}\left(t_{i}\right)-\pi M\left(t_{i}\right)=0 .
\end{aligned}
$$

The parameter values listed in Table 1 were estimated based on some known results and assumptions, taking into consideration the effect of each subgroup/population in the virus spread. Based on official data on the COVID-19 in the UAE among the residents, we consider the estimation of the parameters of the dynamics of the virus. This due to the fact that the dynamics of the virus transmission from a country (e.g., the case study UAE) to another (China) does not change much. In addition, the other parameters are related to the structure of populations and has no affect on the nature of the virus.

The total population $N(0)$ of the UAE in 2019 is approximately 9.666 millions. The life expectancy in the UAE for the year of 2019 is 77.5. Therefore the natural mortality rate is $1 /(77.5 \times 365)$. The birth rate is estimated by multiplying the value of total population times the mortality rate, so it is estimated by the value 341.706 . For the initial values of the model, we consider the population size 9.666 millions for $t=0$. We assume that the number of infected people was 300 and, initially, there were no recovered cases, $R_{p}(0)=0$. Hence we have the following ICs:

$$
S_{p}(0)=\frac{9,344,440}{N_{p}(0)}, \quad A_{p}(0)=\frac{200}{N_{p}(0)}, \quad E_{p}(0)=\frac{321,060}{N_{p}(0)}, \quad M(0)=\frac{5000}{N_{p}(0)}
$$

Now we present some graphical illustrations based on the given parameter vales and simulation of the model given by Equations (3.1)-(3.5). The dynamics of COVID-19 based on different values of $\alpha$ is depicted in Fig. 3. In Figs. 4, 5, and 6, we provide illustrations of 

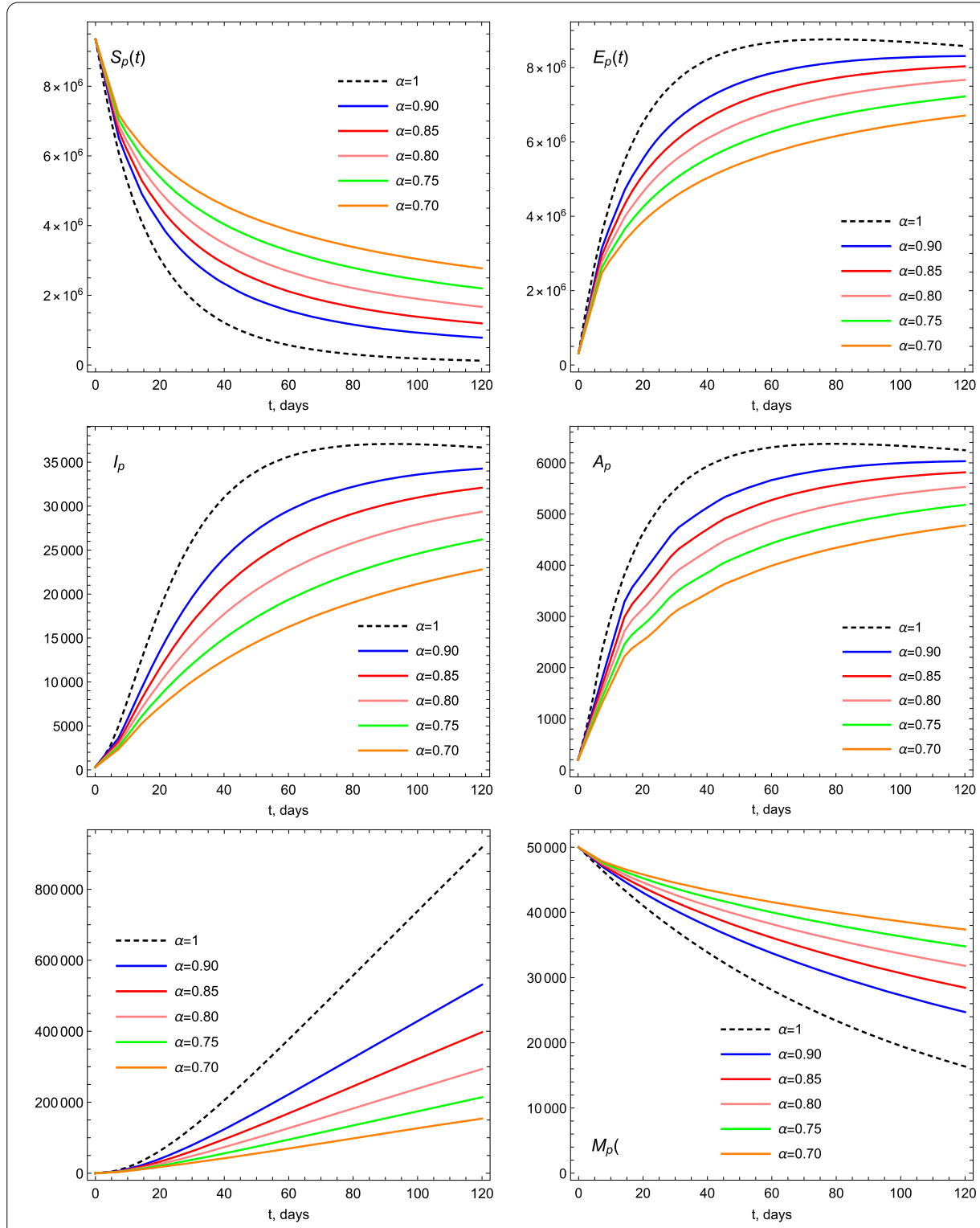

Figure 3 Illustrations of the dynamics of the model parameters using various values of $\alpha$

the stability of the model equilibrium by changing ICs, and we numerically calculate the parameters of the model by considering various ICs of $S_{p}, M$, and $E$.

\section{Conclusion}

In this paper, we initially started the model formulation in terms of the classical integerorder derivative and then apply the Caputo fractional derivative. The model using the $\mathrm{Ca}$ puto fractional derivative best describes the dynamics of the pandemic. The new resulting fractional model of COVID-19 describes the virus dynamics based on the resources and announced cases in the UAE.

We obtained some mathematical results for the model and simulated the original data and fitted it using a new family of Riesz wavelets based on refinable functions that 

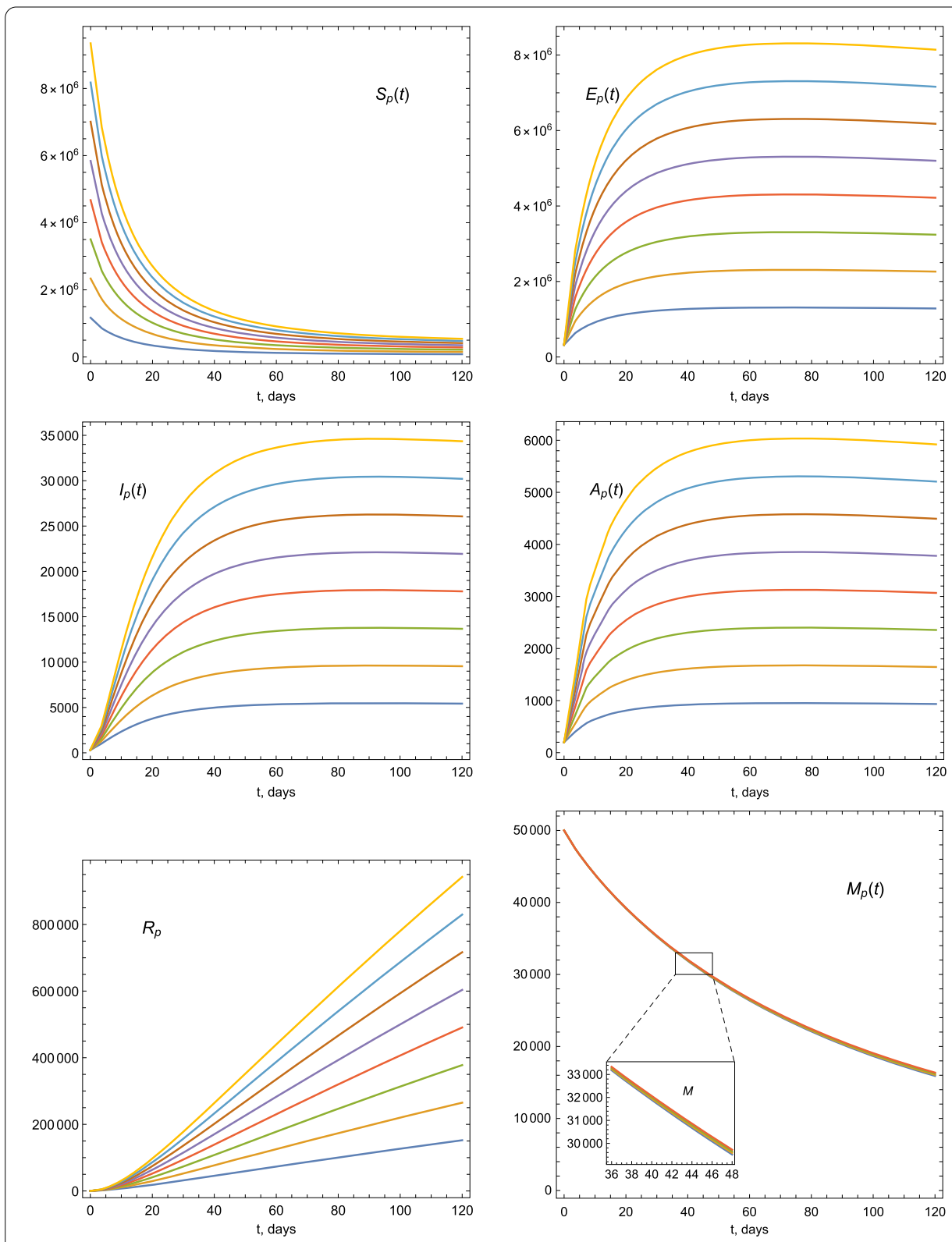

Figure 4 Illustrations of the dynamics of the model parameters using various ICs

have excellent properties such as symmetries and compact support. From the numerical simulation of the fractional model we notice that the fitted data were very accurate compared to the original data and may provide a good start to detect how the virus spreads.

We also provided graphical illustrations (Figs. 3-6) of the model parameters considering different values of fractional order $\alpha$ and various ICs. The presented figures describe the individuals behavior and its stability within the model equilibrium setting. It turns out that decreasing the order results in a decrease of the infection rates. We believe that, the suggested model is suitable to describe the dynamics of this virus and the nature of its 

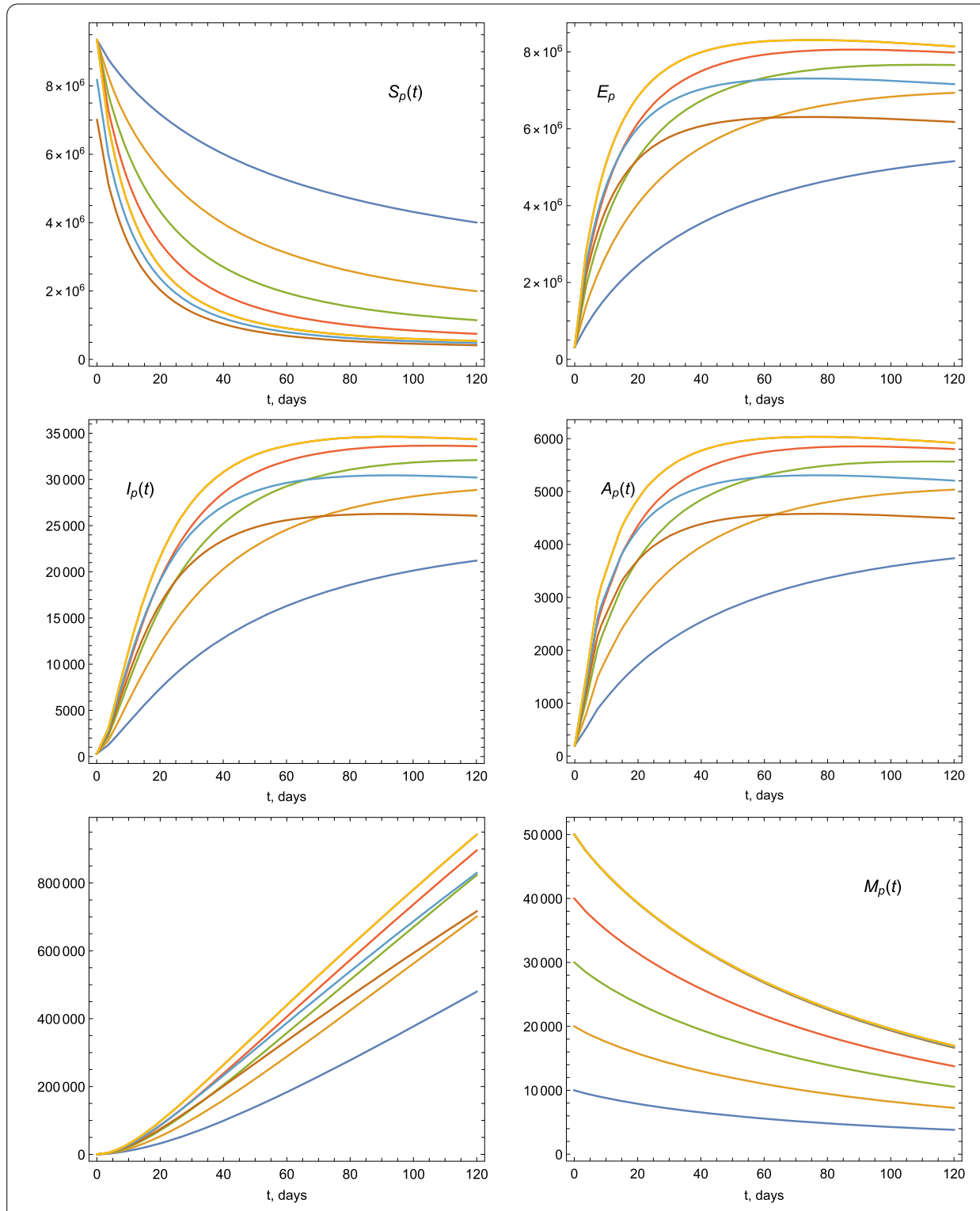

Figure 5 Illustrations of the dynamics of the model parameters using various ICs

spreading. We intended to consider the model with more data resources to better view the dynamics and virus spreading in the country. In future, we will extend the research to include various parameters and aspects, specifically, to predict the virus and positive cases under fitting the data with quarantine and stem cells factors.

In future, we are interested to work on the model presented in [3] by involving a new fractional-order derivative and specifically based on the UAE to further better recognize the dynamics of the new model to provide a feasible solution working successfully, especially for education authorities that are planning future activities. 

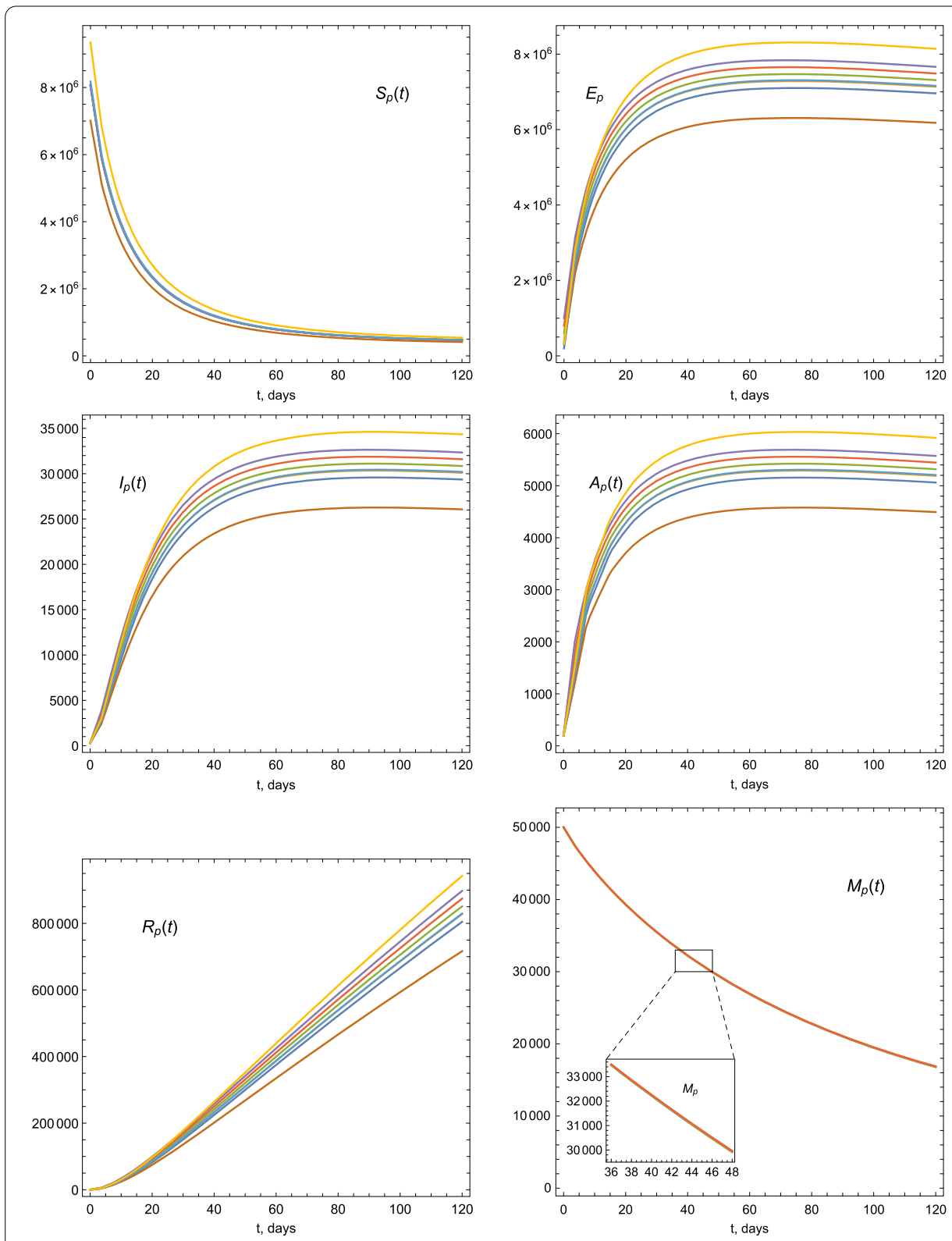

Figure 6 Illustrations of the dynamics of the model parameters using various ICS

\section{Acknowledgements}

We would like to thank the reviewers for their thoughtful comments and efforts toward improving our manuscript.

\section{Funding}

The author(s) received no financial support for this article.

\section{Availability of data and materials}

Not applicable.

\section{Competing interests}

The authors declare that they have no competing interests.

\section{Authors' contributions}

The authors declare that the study was realized in collaboration with a distribution of responsibility. All authors read and approved the final manuscript. 


\section{Author details}

${ }^{1}$ Zayed University, Abu Dhabi, United Arab Emirates. ${ }^{2}$ Kuban State Agrarian University, Krasnodar, Russia. ${ }^{3}$ Engineering School, Viterbo, Italy.

\section{Publisher's Note}

Springer Nature remains neutral with regard to jurisdictional claims in published maps and institutional affiliations.

Received: 15 May 2020 Accepted: 28 January 2021 Published online: 19 February 2021

\section{References}

1. Wuhan, China Population 1950-2020. https://www.macrotrends.net/cities/20712/wuhan/population

2. Is the World Ready for the Coronavirus? Editorial. The New York Times, 2020, Archived from the original on 30 January 2020

3. Gandolfi, A.: Planning of school teaching during Covid-19. Phys. D, Nonlinear Phenom. 415, 132753 (2020). https://doi.org/10.1016/j.physd.2020.132753

4. Ghanbari, B., Atangana, A.: A new application of fractional Atangana-Baleanu derivatives: designing ABC-fractional masks in image processing. Phys. A, Stat. Mech. Appl. 542, 123516 (2020). https://doi.org/10.1016/j.physa.2019.123516

5. Atangana, A., Bonyah, E., Elsadany, A.: A fractional order optimal 4D chaotic financial model with Mittag-Leffler law. Chin. J. Phys. 65, 38-53 (2020). https://doi.org/10.1016/j.cjph.2020.02.003

6. Atangana, A., Aguilar, J., Kolade, M., Hristov, J.: Fractional differential and integral operators with non-singular and non-local kernel with application to nonlinear dynamical systems. Chaos Solitons Fractals 2020, 13 (2020). https://doi.org/10.1016/j.chaos.2019.109493

7. Atangana, A., Baleanu, D.: New fractional derivatives with nonlocal and non-singular kernel: theory and application to heat transfer model. Therm. Sci. 20, 763-769 (2016)

8. Atangana, A., Koca, I.: Chaos in a simple nonlinear system with Atangana-Baleanu derivatives with fractional order. Chaos Solitons Fractals 89, 447-454 (2016)

9. Atangana, A.: On the new fractional derivative and application to nonlinear Fisher's reaction-diffusion equation. Appl. Math. Comput. 273, 948-956 (2016)

10. Atangana, A., Aguilar, J.: Decolonisation of fractional calculus rules: breaking commutativity and associativity to capture more natural phenomena. Eur. Phys. J. Plus 133, 166 (2018)

11. Gao, W., Veeresha, P., Prakasha, P.G., Haci, B.: Novel dynamic structures of 2019-nCoV with nonlocal operator via powerful computational technique. Biology 9(5), 107 (2020). https://doi.org/10.3390/biology9050107

12. Cattani, C.: A review on harmonic wavelets and their fractional extension. J. Adv. Eng. Comput. 2, 224-238 (2018). https://doi.org/10.25073/jaec.201824.225

13. Al-Ghafri, K.S., Rezazadeh, R.: Solitons and other solutions of $(3+1)$-dimensional space-time fractional modified KdV-Zakharov-Kuznetsov equation. Appl. Math. Nonlinear Sci. 4, 289-304 (2019). https://doi.org/10.2478/AMNS.2019.2.00026

14. Singh, J., Kumar, D., Hammouch, Z., Atangana, A.: A fractional epidemiological model for computer viruses pertaining to a new fractional derivative. Appl. Math. Comput. 316, 504-515 (2018). https://doi.org/10.1016/j.amc.2017.08.048

15. Yokus, A., Gulbahar, S.: Numerical solutions with linearization techniques of the fractional Harry Dym equation. Appl. Math. Nonlinear Sci. 4, 35-42 (2019). https://doi.org/10.2478/AMNS.2019.1.00004

16. Khan, M.A., Atangana, A.: Modeling the dynamics of novel coronavirus (2019-nCov) with fractional derivative. Alex. Eng. J. 59(4), 2379-2389 (2020). https://doi.org/10.1016/j.aej.2020.02.033

17. Mohammad, M., Lin, E.B.: Gibbs phenomenon in tight framelet expansions. Commun. Nonlinear Sci. Numer. Simul. 55, 84-92 (2018). https://doi.org/10.1016/j.cnsns.2017.06.029

18. Mohammad, M., Lin, E.B.: Gibbs effects using Daubechies and coiflet tight framelet systems. Contemp. Math. - Am. Math. Soc. 706, 271-282 (2018). https://doi.org/10.1090/conm/706

19. Mohammad, M.: Special B-spline tight framelet and it's applications. J. Adv. Math. Comput. Sci. 29, 1-18 (2018). https://doi.org/10.9734/JAMCS/2018/43716

20. Mohammad, M.: On the Gibbs effect based on the quasi-affine dual tight framelets system generated using the mixed oblique extension principle. Mathematics 7, 952 (2019). https://doi.org/10.3390/math7100952

21. Mohammad, M., Howari, F., Acbas, G., Nazzal, Y., AlAydaroos, F.: Wavelets based simulation and visualization approach for unmixing of hyperspectral data. Int. J. Earth Environ. Sci. 3, Article ID 3:IJEES-152 (2018). https://doi.org/10.15344/2456-351X/2018/152

22. Mohammad, M.: Biorthogonal-wavelet-based method for numerical solution of Volterra integral equations. Entropy 21, 1098 (2019). https://doi.org/10.3390/e21111098

23. Mohammad, M.: A numerical solution of Fredholm integral equations of the second kind based on tight framelets generated by the oblique extension principle. Symmetry 11, 854 (2019). https://doi.org/10.3390/sym11070854

24. Mohammad, M., Cattani, C.: A collocation method via the quasi-affine biorthogonal systems for solving weakly singular type of Volterra-Fredholm integral equations. Alex. Eng. J. 59, 2181-2191 (2020) https://doi.org/10.1016/j.aej.2020.01.046

25. Mohammad, M.: Bi-orthogonal wavelets for investigating Gibbs effects via oblique extension principle. J. Phys. Conf Ser. 1489, 012009 (2020). https://doi.org/10.1088/1742-6596/1489/1/012009

26. Mohammad, M., Cattani, C.: Applications of bi-framelet systems for solving fractional order differential equations. Fractals 28, 2040051 (2020). https://doi.org/10.1142/S0218348X20400514

27. Mohammad, M., Trounev, A.: Implicit Riesz wavelets based-method for solving singular fractional integro-differential equations with applications to hematopoietic stem cell modeling. Chaos Solitons Fractals 138, 109991 (2020). https://doi.org/10.1016/j.chaos.2020.109991

28. Mohammad, M., Trounev, A., Cattani, C.: An efficient method based on framelets for solving fractional Volterra integral equations. Entropy 22, 824 (2020). https://doi.org/10.3390/e22080824 
29. Mohammad, M., Trounev, A.: On the dynamical modeling of Covid-19 involving Atangana-Baleanu fractional derivative and based on Daubechies framelet simulations. Chaos Solitons Fractals 140, 110171 (2020). https://doi.org/10.1016/j.chaos.2020.110171

30. Mohammad, M., Trounev, A.: Fractional nonlinear Volterra-Fredholm integral equations involving Atangana-Baleanu fractional derivative: framelet applications. Adv. Differ. Equ. 2020, 618 (2020). https://doi.org/10.1186/s13662-020-03042-9

31. Daubechies, I., Han, B., Ron, A., Shen, Z.: Framelets: MRA-based constructions of wavelet frames, applied and computational harmonic analysis. Appl. Comput. Harmon. Anal. 14, 1-46 (2003)

32. Selesnick, I.: Smooth wavelet tight frames with zero moments. Appl. Comput. Harmon. Anal. 10, 163-181 (2001)

33. Han, B., Michelle, M.: Construction of wavelets and framelets on a bounded interval. Anal. Appl. 16, 807-849 (2018)

34. Dong, B., Shen, Z.: Pseudo-spline, wavelets and framelets, applied and computational harmonic analysis. Appl. Comput. Harmon. Anal. 22, 78-104 (2007)

35. Li, S., Shen, Y.: Pseudo box splines. Appl. Comput. Harmon. Anal. 26, 344-356 (2008)

36. Dong, B., Dyn, N., Hormann, K.: Properties of dual pseudo-splines. Appl. Comput. Harmon. Anal. 29, 104-110 (2010)

37. Shen, Y., Li, S.: Wavelets and framelets from dual pseudo-splines. Appl. Comput. Harmon. Anal. 54, 1233-1242 (2011)

38. Chuang, Z., Yang, J.: A class of generalized pseudo-splines. J. Inequal. Appl. 2014, 359 (2014)

39. Han, B.: Framelets and Wavelets: Algorithms, Analysis, and Applications. Applied and Numerical Harmonic Analysis. Springer, Cham (2017)

\section{Submit your manuscript to a SpringerOpen ${ }^{\circ}$ journal and benefit from:}

- Convenient online submission

Rigorous peer review

- Open access: articles freely available online

- High visibility within the field

- Retaining the copyright to your article

Submit your next manuscript at $\boldsymbol{~ s p r i n g e r o p e n . c o m ~}$ 